\title{
SECOND-ORDER DEFORMATIONS OF HYDRODYNAMIC-TYPE POISSON BRACKETS
}

\author{
JAMES T. FERGUSON \\ Department of Mathematics, University of Glasgow, Glasgow G12 8QW, UK \\ email: j.ferguson@maths.gla.ac.uk
}

\begin{abstract}
This paper is concerned with the properties of differential-geometrictype Poisson brackets specified by a differential operator of degree 2 . It also considers the conditions required for such a Poisson bracket to form a bi-Hamiltonian structure with a hydrodynamic-type Poisson bracket.
\end{abstract}

1. Introduction. One defines a Poisson bracket on an n-dimensional manifold $M$ as a map $C^{\infty}(M) \times C^{\infty}(M) \rightarrow C^{\infty}(M),(f, g) \mapsto\{f, g\}$, satisfying, for any functions $f, g, h$ on $M$

(1) anti-symmetry: $\{f, g\}=-\{g, f\}$;

(2) linearity: $\{a f+b g, h\}=a\{f, h\}+b\{g, h\}$ for any constants $a, b$;

(3) product rule: $\{f g, h\}=f\{g, h\}+g\{f, h\}$; and

(4) Jacobi identity: $\{\{f, g\}, h\}+\{\{g, h\}, f\}+\{\{h, f\}, g\}=0$.

The first three conditions identify $\{\cdot, \cdot\}$ as a bivector: a rank two, anti-symmetric, contravariant tensor field $\omega$ on $M$. It can therefore be represented, by introducing coordinates $\left\{u^{i}\right\}$ on $M$, as a matrix of coefficients $\omega^{i j}$, giving

$$
\omega=\omega^{i j} \frac{\partial}{\partial u^{i}} \otimes \frac{\partial}{\partial u^{j}}=\frac{1}{2} \omega^{i j} \frac{\partial}{\partial u^{i}} \wedge \frac{\partial}{\partial u^{j}}
$$

and

$$
\{f, g\}=\omega^{i j} \frac{\partial f}{\partial u^{i}} \frac{\partial g}{\partial u^{j}} .
$$

The Jacobi identity places the following constraint on the components of $\omega$ :

$$
\omega^{i r} \frac{\partial \omega^{j k}}{\partial u^{r}}+\omega^{j r} \frac{\partial \omega^{k i}}{\partial u^{r}}+\omega^{k r} \frac{\partial \omega^{i j}}{\partial u^{r}}=0 .
$$

If the matrix $\omega^{i j}$ is non-degenerate, we may introduce its inverse $\omega_{i j}$, satisfying $\omega_{i r} \omega^{r j}=\delta_{i}^{j}$. The Jacobi identity for $\omega^{i j}$ is equivalent to the closedness of $\omega_{i j}$.

One may also introduce Poisson brackets on infinite-dimensional manifolds. The loop space of a finite-dimensional manifold $M, L(M)$, is the space of smooth maps $u: S^{1} \rightarrow M$. Poisson brackets relating Hamiltonians to flows in $L(M)$ will therefore act on functionals mapping $L(M) \rightarrow \mathbb{R}$. In $[\mathbf{4}, \mathbf{5}]$, Dubrovin and Novikov studied the so-called Poisson brackets of differential geometric type, which are of the form

$$
\{f, g\}=\int \frac{\delta f}{\delta u^{i}} P^{i j}\left(\frac{\delta g}{\delta u^{j}}\right) d x,
$$


where $u^{i}$ are coordinates on the target space $M$ and $x$ is the coordinate on $S^{1} . P^{i j}$ is a matrix of differential operators (in $d / d x$ ), with no explicit dependence on $x$, which is assumed to be polynomial in the derivatives $u_{x}^{i}, u_{x x}^{i}, \ldots$ If $\{\cdot, \cdot\}$ defines a Poisson bracket on the loop space, then $P$ is referred to as a Hamiltonian operator.

There is a grading on such operators, preserved by diffeomorphisms of $M$, given by assigning degree 1 to $d / d x$ and degree $n$ to the $n$th $x$-derivative of each field $u^{i}$. An important class is the hydrodynamic-type Poisson brackets, which are homogeneous of degree 1:

$$
P^{i j}=g^{i j}(u) \frac{d}{d x}+\Gamma_{k}^{i j}(u) u_{x}^{k}
$$

According to the programme set out by Novikov [10], differential-geometric-type Poisson brackets on $L(M)$ should be studied in terms of finite-dimensional differential geometry on the target space $M$. When expanded as a polynomial in $d / d x$ and the field derivatives, the coefficients, which are functions of the fields $u^{i}$ alone, can often be naturally related to known objects of differential geometry or else used to define new ones. In the hydrodynamic case, for instance, with $g^{i j}$ non-degenerate, $P$ is Hamiltonian if and only if $g^{i j}$ is a flat metric on $M$, and $\Gamma_{i j}^{k}=-g_{i r} \Gamma_{j}^{r k}$ are the Christoffel symbols of its Levi-Civita connection.

This paper is concerned with Hamiltonian operators which are homogeneous of degree 2. Section 2 describes the geometry of such operators. Section 3 considers the properties of inhomogeneous Hamiltonian operators containing degree 1 and degree 2 parts. We refer the interested reader to [7] for further details and proofs.

\section{Hamiltonian operators of degree 2.}

Definition 2.1. By a Hamiltonian operator of degree 2 we mean a differential operator of the form

$$
P^{i j}=a^{i j}\left(\frac{d}{d x}\right)^{2}+b_{k}^{i j} u_{x}^{k} \frac{d}{d x}+c_{k l}^{i j} u_{x}^{k} u_{x}^{l}+c_{k}^{i j} u_{x x}^{k}
$$

in which the matrix $a^{i j}$ is assumed to be non-degenerate and which defines a Poisson bracket by formula (3). We shall call such an operator an $\mathrm{H}_{2} \mathrm{O}$ for short.

Such operators have been considered already in, for example, $[\mathbf{3}, \mathbf{9}, \mathbf{1 0}, \mathbf{1 2}]$. Amongst the material discussed in these papers there is a conditional Darboux theorem stipulating under what circumstances (4) may be put into constant form by a coordinate transformation. The approach taken in these papers is to consider the operator in a special coordinate system in which the coefficients $c_{k}^{i j}$ and $c_{k l}^{i j}$ both vanish; this is unsuitable for a discussion of bi-Hamiltonian structures, and as such we present the results of this section without the use of special coordinates.

The behaviour of the coefficients $a^{i j}, b_{k}^{i j}, c_{k}^{i j}$ and $c_{k l}^{i j}$ under a change of coordinates $\tilde{u}^{i}=\tilde{u}^{i}\left(u^{p}\right)$ can be determined from the transformation rules for $P^{i j}$. This reveals that $a^{i j}$ transforms as a rank 2 contravariant tensor, whilst $b_{k}^{i j}$ and $c_{k}^{i j}$ behave as

$$
b_{k}^{i j}=-2 a^{i r} \bar{\Gamma}_{r k}^{j}
$$

and

$$
c_{k}^{i j}=-a^{i r} \Gamma_{r k}^{j}
$$


where the objects $\Gamma_{i j}^{k}$ and $\bar{\Gamma}_{i j}^{k}$ are the Christoffel symbols of affine connections. We denote the connection with Christoffel symbols $\Gamma_{i j}^{k}$ by $\nabla$ and denote by $a_{i j}$ the inverse of $a^{i j}$ defined by $a_{i r} a^{r j}=\delta_{i}^{j}$. The final coefficient transforms as

$$
\begin{aligned}
\tilde{c}_{k l}^{i j}= & \frac{\partial \tilde{u}^{i}}{\partial u^{p}} \frac{\partial \tilde{u}^{j}}{\partial u^{q}} \frac{\partial u^{r}}{\partial \tilde{u}^{k}} \frac{\partial u^{s}}{\partial \tilde{u}^{l}} c_{r s}^{p q}+\frac{\partial \tilde{u}^{i}}{\partial u^{p}} \frac{\partial \tilde{u}^{j}}{\partial u^{q}} \frac{\partial^{2} u^{r}}{\partial \tilde{u}^{k} \partial \tilde{u}^{l}} c_{r}^{p q}+\frac{\partial \tilde{u}^{i}}{\partial u^{p}} \frac{\partial^{2} \tilde{u}^{j}}{\partial u^{q} \partial u^{s}} \frac{\partial u^{r}}{\partial \tilde{u}^{(k}} \frac{\partial u^{s}}{\left.\partial \tilde{u}^{l}\right)} b_{r}^{p q} \\
& +\frac{\partial \tilde{u}^{i}}{\partial u^{p}} \frac{\partial^{3} \tilde{u}^{j}}{\partial u^{q} \partial u^{r} \partial u^{s}} \frac{\partial u^{r}}{\partial \tilde{u}^{k}} \frac{\partial u^{s}}{\partial \tilde{u}^{l}} a^{p q}+\frac{\partial \tilde{u}^{i}}{\partial u^{p}} \frac{\partial^{2} \tilde{u}^{j}}{\partial u^{q} \partial u^{r}} \frac{\partial^{2} u^{r}}{\partial \tilde{u}^{k} \partial \tilde{u}^{l}} a^{p q}
\end{aligned}
$$

where the brackets denote symmetrisation, and thus does not correspond to any standard object of differential geometry.

We may now write the condition of skew symmetry and the Jacobi identity in an invariant manner.

THEOREM 2.2. An operator P of the form (4) defines a Poisson bracket by equation (3) if and only if

(a) $a^{i j}=-a^{j i}$,

(b) $\nabla_{k} a^{i j}=b_{k}^{i j}-2 c_{k}^{i j}$,

(c) $a^{i r}\left(b_{r}^{j k}-2 c_{r}^{j k}\right)=a^{k r}\left(b_{r}^{i j}-2 c_{r}^{i j}\right)$,

(d) $\nabla$ is flat (zero torsion, zero curvature),

(e) $c_{k l}^{i j}=c_{(k, l)}^{i j}-a_{p r} c_{(k}^{r i} c_{l)}^{p j}$.

From conditions $(b)$ and $(e)$ of Theorem 2.2 we can see that when $P^{i j}$ is Hamiltonian, the coefficients $c_{k l}^{i j}$ and $b_{k}^{i j}$ are determined by $a^{i j}$ and $c_{k}^{i j}$. Thus we can characterise $\mathrm{H}_{2} \mathrm{Os}$ in terms of just these latter two objects.

COROLlaRY 2.3. There is a one-to-one correspondence between $\mathrm{H}_{2} \mathrm{O}$ s on $L(M)$ and pairs $(a, \nabla)$ on $M$ consisting of a non-degenerate bivector $a^{i j}$ and a torsion-free connection $\nabla$ satisfying two conditions: firstly, the curvature of $\nabla$ vanishes, and secondly,

$$
a^{i r} \nabla_{r} a^{j k}=a^{j r} \nabla_{r} a^{k i}
$$

The Christoffel symbols, $\Gamma_{i j}^{k}$, of $\nabla$ are related to $c_{k}^{i j}$ by $c_{k}^{i j}=-a^{i r} \Gamma_{r k}^{j}$. We then have

$$
\begin{aligned}
b_{k}^{i j} & =\nabla_{k} a^{i j}+2 c_{k}^{i j}, \\
c_{k l}^{i j} & =c_{(k, l)}^{i j}-a_{p r} c_{(k}^{r i} c_{l)}^{p j} .
\end{aligned}
$$

If we consider, as a simple case, an operator (4) in which the coefficients $c_{k}^{i j}$ are constant and $\nabla_{k} a^{i j}=0$, and if we allow $b_{k}^{i j}$ and $c_{k l}^{i j}$ to be defined, from $a^{i j}$ and $c_{k}^{i j}$ according to Corollary 2.3, then we have $a^{i j}=A_{k}^{i j} u^{k}+A_{0}^{i j}$, where $A_{k}^{i j}, A_{0}^{i j}$ are constants with $A_{k}^{i j}=c_{k}^{i j}-c_{k}^{j i}$, and the operator is Hamiltonian if and only if

$$
\begin{aligned}
c_{r}^{i j} c_{l}^{r k} & =-c_{r}^{i k} c_{l}^{r j}, \\
A_{l}^{i r} c_{r}^{j k} & =A_{l}^{j r} c_{r}^{i k} \\
\text { and } \quad A_{0}^{i r} c_{r}^{j k} & =A_{0}^{j r} c_{r}^{i k} .
\end{aligned}
$$


If we take an algebra $\mathcal{A}$ with basis $\left\{e^{1}, \ldots, e^{n}\right\}, n=\operatorname{dimM}$, and use $c_{k}^{i j}$ and $A_{0}^{i j}$ to define a multiplication, $\circ$, and skew-symmetric bilinear form, $\langle\cdot, \cdot\rangle$, by

$$
e^{i} \circ e^{j}=c_{r}^{i j} e^{r}
$$

and

$$
\left\langle e^{i}, e^{j}\right\rangle=A_{0}^{i j}
$$

then we may rewrite equations (6) as

$$
\begin{aligned}
(I \circ J) \circ K & =-(I \circ K) \circ J, \\
\Lambda(I, J, K) & =\Lambda(J, I, K) \text { and } \\
\langle I, J \circ K\rangle & =\langle J, I \circ K\rangle,
\end{aligned}
$$

for all $I, J, K \in \mathcal{A}$, where $\Lambda$ is the associator of $\circ: \Lambda(I, J, K)=(I \circ J) \circ K-I \circ(J \circ K)$.

Algebras satisfying conditions (7) and (8) have appeared before in [13], in the context of linear hydrodynamic Hamiltonian operators taking values in a completely odd superspace, where the following definition was proposed.

Definition 2.4. An algebra $(\mathcal{A}, \circ)$ satisfying conditions $(7)$ and $(8)$ is called a Fermionic Novikov algebra.

In [1] Fermionic Novikov algebras in dimensions 2-5 were studied, and the listing therein provides a source of examples of Hamiltonian operators of degree two.

EXAMPLE 2.5.

$$
\begin{aligned}
P & =\left(\begin{array}{cccc}
0 & 0 & 0 & a \\
0 & 0 & -a & -b-(t-1) u^{1} \\
0 & a & 0 & c-u^{2} \\
-a & b+(t-1) u^{1} & -c+u^{2} & 0
\end{array}\right)\left(\frac{d}{d x}\right)^{2} \\
& +2\left(\begin{array}{cccc}
0 & 0 & 0 & 0 \\
0 & 0 & 0 & u_{x}^{1} \\
0 & 0 & -u_{x}^{1} & 0 \\
0 & \tau u_{x}^{1} & u_{x}^{2} & u_{x}^{3}
\end{array}\right)\left(\frac{d}{d x}\right)+\left(\frac{1}{a}\right)\left(\begin{array}{cccc}
0 & 0 & 0 & 0 \\
0 & 0 & 0 & 0 \\
0 & 0 & 0 & \left(u_{x}^{1}\right)^{2} \\
0 & 0 & -\left(u_{x}^{1}\right)^{2} & 0
\end{array}\right) \\
& +\left(\begin{array}{cccc}
0 & 0 & 0 & 0 \\
0 & 0 & 0 & u_{x}^{1} \\
0 & 0 & -u_{x x}^{1} & 0 \\
0 & \tau u_{x x}^{1} & u_{x x}^{2} & u_{x x}^{3}
\end{array}\right)
\end{aligned}
$$

is Hamiltonian for all values of the constants $a, b, c$ and $\tau$ with $a \neq 0$. This is the most general Hamiltonian operator associated in the manner discussed above to the algebra designated $(44)_{\tau}$ in $[\mathbf{1}]$. 
The Darboux theorem for $\mathrm{H}_{2} \mathrm{Os}$ is given in [12] and states that $P$ can be put in the constant form

$$
P^{i j}=\tilde{a}^{i j}\left(\frac{d}{d x}\right)^{2},
$$

where $\tilde{a}^{i j}$ is constant, if and only if the two form $a_{i j}$ inverse to $a^{i j}$ is closed. The following result elucidates this somewhat.

Corollary 2.6. For an $\mathrm{H}_{2} \mathrm{O}$, the following three statements, presented in both covariant and contravariant forms, are equivalent:

(1) The two-form $a_{i j}$ is closed (and so symplectic), or equivalently $a^{i j}$ satisfies equation (2) (and so defines a Poisson bracket on $M$ by equation (1));

(2) $\nabla_{k} a^{i j}=0$, i.e. $\nabla_{k} a_{i j}=0$;

(3) $b_{k}^{i j}=2 c_{k}^{i j}$, i.e. $\Gamma_{i j}^{k}=\bar{\Gamma}_{i j}^{k}$.

Proof. We see, from the characterisation of $\mathrm{H}_{2} \mathrm{Os}$ given in Corollary 2.3,

$$
\begin{aligned}
a^{i j} \text { is Poisson } & \Longleftrightarrow a^{i r} a_{, r}^{j k}+a^{j r} a_{, r}^{k i}+a^{k r} a_{, r}^{i j}=0 \\
& \Longleftrightarrow a^{i r} \nabla_{r} a^{j k}+a^{j r} \nabla_{r} a^{k i}+a^{k r} \nabla_{r} a^{i j}=0 \\
& \Longleftrightarrow 3 a^{k r} \nabla_{r} a^{i j}=0 \\
& \Longleftrightarrow \nabla_{k} a^{i j}=0, \\
& \Longleftrightarrow b_{k}^{i j}=2 c_{k}^{i j} .
\end{aligned}
$$

This means that if the $\mathrm{H}_{2} \mathrm{O}$ can be put into a constant form, then the pair $(a, \nabla)$ associated with it by Corollary 2.3 can be replaced by a pair $(\omega, \nabla)$ consisting of a symplectic form $\omega$ (the inverse of $a^{i j}$ ) and a flat connection $\nabla$ satisfying $\nabla \omega=0$, i.e. a flat symplectic connection (see, for example, [2]). In [7] such a pair $(\omega, \nabla)$ was referred to as a Fedosov structure.

3. Second-order deformations of hydrodynamic type poisson brackets. We now consider a pair of operators, $P_{1}$ and $P_{2}$ in which $P_{1}$ is a Hamiltonian operator of hydrodynamic type and $P_{2}$ is of second order, i.e.

$$
\begin{aligned}
& P_{1}^{i j}=g^{i j} \frac{d}{d x}+\Gamma_{k}^{i j} u_{x}^{k}, \\
& P_{2}^{i j}=a^{i j}\left(\frac{d}{d x}\right)^{2}+b_{k}^{i j} u_{x}^{k} \frac{d}{d x}+c_{k l}^{i j} u_{x}^{k} u_{x}^{l}+c_{k}^{i j} u_{x x}^{k},
\end{aligned}
$$

where $g^{i j}$ is the inverse of a flat metric $g_{i j}$ on $M$ and $\Gamma_{k}^{i j}=-g^{i r} \Gamma_{r k}^{j}$, where the $\Gamma_{i j}^{k}$ are the Christoffel symbols ${ }^{1}$ of the Levi-Civita connection of $g$. We also assume that $P_{2}^{i j}$ is anti-symmetric, so that $a^{i j}=-a^{j i}, b_{k}^{i j}=a_{, k}^{i j}+c_{k}^{i j}+c_{k}^{j i}$ and $c_{k l}^{(i j)}=c_{(k, l)}^{(i j)}$. (We do not assume $P_{2}^{i j}$ satisfies the Jacobi identity.)

The motivation [6] for studying such pairs of operators comes not from regarding them as separate Hamiltonian operators but from thinking of $P_{2}^{i j}$ as a first-order

\footnotetext{
${ }^{1}$ In the previous section we used $\Gamma_{i j}^{k}$ for the Christoffel symbols associated with $c_{k}^{i j}$; in this section $\Gamma_{i j}^{k}$ and $\Gamma_{k}^{i j}$ will always refer to the Levi-Civita connection of $g$.
} 
(dispersive) deformation of $P_{1}^{i j}$ into some non-homogeneous Hamiltonian operator $P^{i j}=P_{1}^{i j}+\varepsilon P_{2}^{i j}+O\left(\varepsilon^{2}\right)$. Thus, in such a pair, it is sensible to regard the geometry of $P_{1}^{i j}$ as being more intrinsic than any associated to $P_{2}^{i j}$.

We choose to work in flat coordinates for $g$ so that $g^{i j}$ is constant and $\Gamma_{k}^{i j}=0$. Direct calculation of the Jacobi identity for $P^{i j}$ in these coordinates yields the following.

THEOREM 3.1. $P_{2}$ is an infinitesimal deformation of $P_{1}$; i.e. $P^{i j}=P_{1}^{i j}+\varepsilon P_{2}^{i j}+O\left(\varepsilon^{2}\right)$ satisfies the Jacobi identity to order $\varepsilon$ if and only if

(a) $g^{i r} c_{r}^{j k}+g^{j r} c_{r}^{i k}=0$

(b) $c_{k l}^{i j}=c_{(k, l)}^{i j}$,

(c) $g^{i r} c_{l, r}^{j k}=g^{j r}\left(c_{l, r}^{i k}-c_{r, l}^{i k}\right)$ and

(d) $g^{i r}\left(a_{, r}^{j k}-c_{r}^{j k}\right)+g^{j r}\left(a_{, r}^{k i}-c_{r}^{k i}\right)+g^{k r}\left(a_{, r}^{i j}-c_{r}^{i j}\right)=0$

in the flat coordinates for $g^{i j}$.

PROPOSITION 3.2. If $P_{2}$ is an infinitesimal deformation of $P_{1}$, then there exists a tensor field $A_{j}^{i}$ such that

$$
\begin{aligned}
a^{i j} & =g^{i r} A_{r}^{j}-g^{j r} A_{r}^{i}, \\
b_{k}^{i j} & =2 g^{i s} A_{s, k}^{j}-g^{i r} A_{k, r}^{i}-g^{i s} A_{k, s}^{j}, \\
c_{k l}^{i j} & =g^{i s} A_{s, k l}^{j}-g^{i s} A_{(k, l) s}^{j}, \\
c_{k}^{i j} & =g^{i s} A_{s, k}^{j}-g^{i s} A_{k, s}^{j}
\end{aligned}
$$

in flat coordinates for $g^{i j}$. Further, any (1,1)-tensor field $A_{j}^{i}$ produces an infinitesimal deformation of $P_{1}$ by the above formulae.

Proposition 3.2 may be understood as asserting the existence of an evolutionary vector field

$$
e=A_{j}^{i}(u(x)) u_{x}^{j}(x) \frac{\partial}{\partial u^{i}(x)}+\cdots,
$$

satisfying $P_{2}=-\mathcal{L}_{e} P_{1}$ whenever $P_{2}$ is an infinitesimal deformation of $P_{1}$. This is therefore not a surprising result; in [8] Getzler showed the triviality of infinitesimal deformations of Hydrodynamic-type Poisson brackets.

There is a freedom in $A_{j}^{i}$ of $A_{j}^{i} \mapsto A_{j}^{i}+g^{i r} f_{, j}$ for some function $f$, which does not affect the coefficients of $P_{2}$. This corresponds to adjusting $e$ by a Hamiltonian vector field, $e \mapsto e+P_{1}(\delta f)$.

If, with reference to Corollary 2.6, we impose the additional constraint on (10) that $b_{k}^{i j}=2 c_{k}^{i j}$, then we have the potentiality condition $g_{j r} A_{k, i}^{r}=g_{i r} A_{k, j}^{r}$, so that there exists a one-form $B_{k}$ such that

$$
A_{j}^{i}=g^{i r} B_{j, r}
$$

In this case $a^{i j}=g^{i r} g^{j r}\left(B_{r, s}-B_{s, r}\right)=g^{i r} g^{j r}(d B)_{r s}$; so $a^{i j}$ and $g^{i j}$ together define the exterior derivative of $B$. This means that they define $B$ to within the exterior derivative of a function; however, the freedom $B \mapsto B+d f$ corresponds to changing $A_{j}^{i}$ to $A_{j}^{i}+g^{i r} f_{, r j}$ and as such has no effect on the coefficients of $P_{2}$. So, given $a^{i j}$ and $g^{i j}$, if we wish to 
have $b_{k}^{i j}=2 c_{k}^{i j}$, then $c_{k}^{i j}$ and $c_{k l}^{i j}$ are determined uniquely. In fact we may write explicitly

$$
c_{k}^{i j}=g^{j s} g_{k r} \frac{\partial a^{i r}}{\partial u^{s}}, \quad c_{k l}^{i j}=c_{(k, l)}^{i j},
$$

and with this, $P_{2}$ is an infinitesimal deformation of $P_{1}$ if and only if

$$
g^{i r} a_{, r}^{j k}+g^{j r} a_{, r}^{k i}+g^{k r} a_{, r}^{i j}=0
$$

which is equivalent to the closedness of the two-form

$$
\phi_{i j}=g_{i r} g_{j s} a^{r s} \text {. }
$$

COROLLARY 3.3. Given a flat metric $g$ and a symplectic form $\omega$, there is at most one choice of flat symplectic connection $\nabla$ such that the $\mathrm{H}_{2} \mathrm{O}$ specified by $(\omega, \nabla)$ is compatible with the hydrodynamic operator specified by $g$.

One must check that the operator

$$
P_{2}^{i j}=\omega^{i j}\left(\frac{d}{d x}\right)^{2}+2 c_{k}^{i j} u_{x}^{k} \frac{d}{d x}+c_{k l}^{i j} u_{x}^{k} u_{x}^{l}+c_{k}^{i j} u_{x x}^{k}
$$

with coefficients given by substituting $\omega^{i j}$ into (12) is Hamiltonian by Theorem 2.2. If it is, then compatibility with $P_{1}$ will follow immediately, since equation (13) is a consequence of the anti-symmetry of $P_{2}$.

We conclude this section with an example of this type.

EXAMPLE 3.4. The Kaup-Broer system [11],

$$
\left(\begin{array}{l}
u_{t}^{1} \\
u_{t}^{2}
\end{array}\right)=\left(\begin{array}{c}
u_{x x}^{1}+2 u_{x}^{2}+2 u^{1} u_{x}^{1} \\
-u_{x x}^{2}+2\left(u^{1} u^{2}\right)_{x}
\end{array}\right)
$$

is described by the pair of compatible Hamiltonian operators

$$
\begin{aligned}
& P_{1}=\left(\begin{array}{ll}
0 & 1 \\
1 & 0
\end{array}\right) \frac{d}{d x} \\
& P_{2}=\left(\begin{array}{cc}
0 & 1 \\
-1 & 0
\end{array}\right)\left(\frac{d}{d x}\right)^{2}+\left(\begin{array}{cc}
2 & u^{1} \\
u^{1} & 2 u^{2}
\end{array}\right) \frac{d}{d x}+\left(\begin{array}{ll}
0 & u_{x}^{1} \\
0 & u_{x}^{2}
\end{array}\right)
\end{aligned}
$$

Scaling $x \mapsto \varepsilon x, t \mapsto \varepsilon t$ splits $P_{2}$ into $P_{2}^{(1)}+\varepsilon P_{2}^{(2)}$, where

$$
\begin{aligned}
& P_{2}^{(1)}=\left(\begin{array}{cc}
2 & u^{1} \\
u^{1} & 2 u^{2}
\end{array}\right) \frac{d}{d x}+\left(\begin{array}{ll}
0 & u_{x}^{1} \\
0 & u_{x}^{2}
\end{array}\right) \\
& P_{2}^{(2)}=\left(\begin{array}{cc}
0 & 1 \\
-1 & 0
\end{array}\right)\left(\frac{d}{d x}\right)^{2}
\end{aligned}
$$

Since $P_{2}=P_{2}^{(1)}+\varepsilon P_{2}^{(2)}$ is Hamiltonian for all $\varepsilon, P_{2}^{(1)}$ and $P_{2}^{(2)}$ constitute a biHamiltonian structure of the type considered above. A set of flat coordinates for 
the metric in $P_{2}^{(1)}$ are

$$
\begin{aligned}
& \tilde{u}^{1}=u^{1}, \\
& \tilde{u}^{2}=\sqrt{4 u^{2}-\left(u^{1}\right)^{2}},
\end{aligned}
$$

in which

$$
\begin{aligned}
\tilde{P}_{2}^{(1)}= & \left(\begin{array}{ll}
2 & 0 \\
0 & 2
\end{array}\right) \frac{d}{d x}, \\
\tilde{P}_{2}^{(2)}= & \frac{2}{\tilde{u}^{2}}\left(\begin{array}{cc}
0 & 1 \\
-1 & 0
\end{array}\right)\left(\frac{d}{d x}\right)^{2}+\frac{4}{\left(\tilde{u}^{2}\right)^{2}}\left(\begin{array}{cc}
0 & -\tilde{u}_{x}^{2} \\
0 & \tilde{u}_{x}^{1}
\end{array}\right) \frac{d}{d x} \\
& +\frac{4}{\left(\tilde{u}^{2}\right)^{3}}\left(\begin{array}{cc}
0 & \left(\tilde{u}_{x}^{2}\right)^{2} \\
0 & -\tilde{u}_{x}^{1} \tilde{u}_{x}^{2}
\end{array}\right)+\frac{2}{\left(\tilde{u}^{2}\right)^{2}}\left(\begin{array}{cc}
0 & -\tilde{u}_{x x}^{2} \\
0 & \tilde{u}_{x x}^{1}
\end{array}\right) .
\end{aligned}
$$

So in this situation we have, for the one-form in (11),

$$
B=\frac{\tilde{u}^{1}}{2 \tilde{u}^{2}} d \tilde{u}^{2}
$$

ACKNOWLEDGEMENTS. The author would like to thank Ian Strachan for suggesting this project and the Carnegie Trust for the Universities of Scotland for the scholarship under which this work was conducted.

\section{REFERENCES}

1. C. Bai, D. Meng and L. He, On Fermionic Novikov algebras, J. Phys. A 35 (47) (2002), 10053-10063.

2. P. Bieliavsky, M. Cahen, S. Gutt, J. Rawnsley and L. Schwachhöfer, Symplectic connections, Int. J. Geom. Methods Mod. Phys. 3(3) (2006), 375-420.

3. P. W. Doyle, Differential geometric Poisson bivectors in one space variable, J. Math. Phys. 34(4) (1993), 1314-1338.

4. B. A. Dubrovin and S. P. Novikov, Poisson brackets of hydrodynamic type, Dokl. Akad. Nauk SSSR, 279(2) (1984), 294-297.

5. B. A. Dubrovin and S. P. Novikov, Hydrodynamics of weakly deformed soliton lattices: Differential geometry and Hamiltonian theory, Uspekhi Mat. Nauk 44(6(270)) (1989), 29-98, 203.

6. B. A. Dubrovin and Y. Zhang, Normal forms of hierarchies of integrable pde's, Frobenius manifolds and Gromov-Witten invariants. arXiv.org:math.DG/0108160, 2001.

7. J. T. Ferguson, Flat pencils of symplectic connections and Hamiltonian operators of degree 2. J. Geom. Phys. 58(4) (2008), 468-486.

8. E. Getzler, A Darboux theorem for Hamiltonian operators in the formal calculus of variations. Duke Math. J. 111(3) (2002), 535-560.

9. O. I. Mokhov, Symplectic and Poisson structures on loops spaces of smooth manifolds, and integrable systems. Russian Mathematical Surveys 53(3) (1998), 515-622.

10. S. P. Novikov, The geometry of conservative systems of hydrodynamic type. the method of averaging for field-theoretical systems. Russian Mathematical Surveys, 40(4) (1985), 85-98.

11. W. Oevel, A note on the Poisson brackets associated with Lax operators. Phys. Lett. A 186(1-2) (1994), 79-86.

12. G. V. Potëmin, Poisson brackets of differential-geometric type. Dokl. Akad. Nauk SSSR, 286(1) (1986), 39-42.

13. X. Xu, Variational calculus of supervariables and related algebraic structures. J. Algebra, 223(2) (2000), 396-437. 